Full length article

\title{
Cross-linking of a biopolymer-peptide co-assembling system
}

\author{
Karla E. Inostroza-Brito a,b,1, Estelle C. Collin ${ }^{\mathrm{a}, \mathrm{b}, 1}$, Anna Majkowska ${ }^{\mathrm{a}, \mathrm{b}}$, Sherif Elsharkawy ${ }^{\mathrm{a}, \mathrm{b}}$, Alistair Rice ${ }^{\mathrm{c}}$, \\ Armando E. del Río Hernández ${ }^{c}$, Xin Xiao ${ }^{a}$, José Rodríguez-Cabello ${ }^{d}$, Alvaro Mata ${ }^{\mathrm{a}, \mathrm{b}, *}$ \\ a Institute of Bioengineering, Queen Mary University of London, London E1 4NS, UK \\ ${ }^{\mathrm{b}}$ School of Engineering \&' Materials Science, Queen Mary University of London, London E1 4NS, UK \\ ${ }^{\mathrm{c}}$ Cellular and Molecular Biomechanics Laboratory, Department of Bioengineering, Imperial College London, SW7 2AZ, UK \\ ${ }^{\mathrm{d}}$ BIOFORGE Lab, University of Valladolid, CIBER-BBN, Valladolid 47011, Spain
}

\section{A R T I C L E I N F O}

\section{Article history:}

Received 9 February 2017

Received in revised form 26 April 2017

Accepted 17 May 2017

Available online $\mathrm{xxxx}$

\section{Keywords:}

Cross-linking

Dynamic biomaterials

Tissue engineering

Self-assembly

Elastin-like recombinamer (ELR)

\begin{abstract}
A B S T R A C T
The ability to guide molecular self-assembly at the nanoscale into complex macroscopic structures could enable the development of functional synthetic materials that exhibit properties of natural tissues such as hierarchy, adaptability, and self-healing. However, the stability and structural integrity of these kinds of materials remains a challenge for many practical applications. We have recently developed a dynamic biopolymer-peptide co-assembly system with the capacity to grow and undergo morphogenesis into complex shapes. Here we explored the potential of different synthetic (succinimidyl carboxymethyl ester, poly (ethylene glycol) ether tetrasuccinimidyl glutarate and glutaraldehyde) and natural (genipin) crosslinking agents to stabilize membranes made from these biopolymer-peptide co-assemblies. We investigated the cross-linking efficiency, resistance to enzymatic degradation, and mechanical properties of the different cross-linked membranes. We also compared their biocompatibility by assessing the metabolic activity and morphology of adipose-derived stem cells (ADSC) cultured on the different membranes. While all cross-linkers successfully stabilized the system under physiological conditions, membranes cross-linked with genipin exhibited better resistance in physiological environments, improved stability under enzymatic degradation, and a higher degree of in vitro cytocompatibility compared to the other cross-linking agents. The results demonstrated that genipin is an attractive candidate to provide functional structural stability to complex self-assembling structures for potential tissue engineering or in vitro model applications.
\end{abstract}

\section{Statement of Significance}

Molecular self-assembly is widely used for the fabrication of complex functional biomaterials to replace and/or repair any tissue or organ in the body. However, maintaining the stability and corresponding functionality of these kinds of materials in physiological conditions remains a challenge. Chemical crosslinking strategies (natural or synthetic) have been used in an effort to improve their structural integrity. Here we investigate key performance parameters of different cross-linking strategies for stabilising selfassembled materials with potential biomedical applications using a novel protein-peptide co-assembling membrane as proof-of-concept. From the different cross-linkers tested, the natural cross-linker genipin exhibited the best performance. This cross-linker successfully enhanced the mechanical properties of the system enabling the maintenance of the structure in physiological conditions without compromising its bioactivity and biocompatibility. Altogether, we provide a systematic characterization of cross-linking alternatives for self-assembling materials focused on biocompatibility and stability and demonstrate that genipin is a promising alternative for the cross-linking of such materials with a wide variety of potential applications such as in tissue engineering and drug delivery.

(C) 2017 Acta Materialia Inc. Published by Elsevier Ltd. All rights reserved.

\footnotetext{
* Corresponding author at: School of Engineering \& Materials Science, Queen Mary University of London, London E1 4NS, UK.

E-mail address: a.mata@qmul.ac.uk (A. Mata).

${ }^{1}$ Same contribution authors.
}

\section{Introduction}

In biological systems, molecules can tidily bind one to another by non-covalent interactions (e.g. hydrogen bonds, van der Waals, 
electrostatic and hydrophobic interactions), forming complex hierarchical structures with outstanding functions. The last two decades have seen a remarkable increase in studies aiming to exploit supramolecular chemistry for the fabrication of materials that attempt to recreate the molecular organization found in Nature [1]. A wide variety of natural and synthetic molecules are being used as self-assembling building-blocks of hydrogel materials with promising bioactive and mechanical properties [2-4] for applications in bioactive surfaces [5], devices [6], and implants [7].

While the potential of self-assembling biomaterials has been well demonstrated $[1,8]$, guiding molecular organization through non-covalent interactions across scales in order to recreate the structural complexity and functionality of natural tissues has been challenging [9]. An increasing number of strategies aim to fabricate, through molecular self-assembly, hierarchical structures with precision and tuneability [1,3,9-12]. Our group has recently reported a co-assembling system based on the conformational modification of elastin-like recombinamers (ELRs) by peptide amphiphiles (PAs) that enables the fabrication of membranes with the capacity to undergo morphogenesis into complex tubular micro/macro structures [10]. When a drop of PA is injected into a larger volume of ELP, an interfacial assembly spontaneously develops, forming a membrane that opens and adheres upon contact to any surface. The process leads to the formation of tubular membranes that display controlled assembly and disassembly capabilities, adhesion and sealing to surfaces, self-healing and the capability to undergo morphogenesis into complex structures with high spatiotemporal control (Fig. 1B). However, as most selfassembled biomaterials [13], these membranes lack the mechanical properties required for many biomedical applications due to the nature of non-covalent interactions involved in the selfassembly of molecules, which limits their applicability. Indeed, hydrogen bonds have very low association strength in hydrogels due to competition of water for binding sites, hydrophobic interactions are highly limited by solubility of the hydrophobes in solution [14], and hydrophilic polymers tend to dissolve into the aqueous phase [15]. All these phenomena tend to weaken the hydrogel network. Therefore, there is a need for cross-linking strategies that can enhance the mechanical properties of these materials in a practical and biocompatible manner in order to maximize the benefits of the self-assembling process such as bioactivity, complexity, and structural hierarchy.

Different chemical, biological, and physical cross-linking strategies have been proposed to increase the mechanical properties of self-assembled materials $[13,16]$. The ideal cross-linker should be capable to covalently link molecular groups of these buildingblocks without compromising their hierarchical order, or eliciting cytotoxic, or immune reactions [17]. Natural and chemical crosslinkers can therefore enhance hydrogel stability and mechanical performance by introducing covalent bonds within the hydrogel, preventing the dissolution of the molecules into the aqueous phase.

Glutaraldehyde (GTA) has been widely used to cross-link biomaterials due to its efficacy in enhancing their mechanical properties [17]. However, GTA has been associated with dystrophic calcifications, pro-inflammatory response by activation of macrophage-like cells, and cytotoxic effects when used to crosslink implanted materials $[16,17]$. Therefore, cross-linking strategies based on multifunctional poly (ethylene glycol) (PEG) systems and plant extracts have been proposed as alternative cross-linker candidates. Multifunctional PEG cross-linking systems are either linear or exhibit branched chains with terminal active ester groups that react with the primary amine groups of the building blocks $[13,18]$. Poly (ethylene glycol) ether tetrasuccinimidyl glutarate (4S-StarPEG), a multi-armed functional PEG molecule, has been reported as an effective cross-linker of hydrogels [18,19] and implantable fibres [13]. The resulting scaffolds demonstrated improved stability and mechanical properties in physiological environments while exhibiting good biocompatibility [18,19]. Similarly, succinimidyl carboxymethyl ester (SCM-PEG-SCM), a linear functionalised PEG cross-linker, has been shown to improve stability and mechanical properties of collagen hydrogels with good biocompatibility $[17,20]$. Natural molecules from plant extracts have also been used as cross-linking agents for decades to enhance stability of materials for biomedical applications. Genipin (GNP), a natural cross-linker agent derived from the fruit of Gardenia jasminoides Ellis, reacts with primary amine groups of the building blocks via its hydroxyl and carboxyl reactive groups [21,22]. GNP has been shown to exhibit lower cytotoxicity and inflammatory responses compared to GTA, and to possess antiphlogistic, antiinflammatory, diuretic, choleretic, and haemostatic properties [23].

In this study, we investigated possible exogenous cross-linking strategies (4S-StarPEG, SCM-PEG-SCM, GNP, and GTA) for the enhancement of stability and structural integrity of selfassembling systems with potential biomedical application. Specifically, we explored the performance of these cross-linkers in the biopolymer-peptide self-assembling system (ELR/PA membrane) previously reported by our group as ELP/PA system [10]. We compared cross-linking efficiency, resistance to enzymatic degradation, and potential toxicity of these cross-linkers, in order to identify an effective cross-linker agent that does not compromise hierarchical nano/microstructure and bioactive properties.

\section{Experimental section}

\subsection{Materials}

Peptide amphiphile (PA) molecules $\left(\mathrm{C}_{15} \mathrm{H}_{31} \mathrm{CONH}-\mathrm{VVVAAAKKK}-\right.$ $\mathrm{CONH}_{2}$ ) were obtained from Cambridge peptides (Birmingham, UK). Elastin-like recombinamers (ELR) molecules (MESLLP[(VPGVG VPGVG VPGEG VPGVG VPGVG) $\left.10^{-}(\mathrm{VGIPG})_{60}\right]_{2^{-}}$ [(VPGIG) ${ }_{10}$-AVTGRGDSPASS(VPGIG) $\left.\left.)_{10}\right]_{2}-\mathrm{V}\right)$ were provided by Technical Proteins NBT S.L. (Valladolid, Spain). Glutaraldehyde (GTA), collagenase $(\mathrm{Cg})$, pronase $(\mathrm{Pn})$, proteinase $\mathrm{K}(\mathrm{PK})$ and trypsin $(\mathrm{Tp})$ were purchased from Sigma Aldrich (Dorset, UK). Genipin (GNP) was purchased from Cambridge Bioscience (Cambridge, UK). Succinimidyl carboxymethyl ester (SCM-PEG-SCM, molecular weight $2000 \mathrm{Da}$ ) and poly (ethylene glycol) ether tetrasuccinimidyl glutarate (4S-StarPEG, molecular weight 10,000 Da) were purchased from JenKem Technology (Texas, USA). Elastase (Et) was purchased from Elastin Products Company (Missouri, USA). 2,4,6 Trinitrobenzenesulfonic acid (TNBS) assay was purchased from Thermo Scientific $^{\mathrm{TM}}$ Pierce $^{\mathrm{TM}}$ (Paisley, UK).

\subsection{ELR/PA membrane fabrication}

ELR and PA molecules were dissolved in ultrapure water to a final concentration of $0.1 \mathrm{mM}$ and $8.7 \mathrm{mM}$, respectively. $10 \mu \mathrm{l}$ drop of PA solution was added in $190 \mu$ l of ELR solution (Fig. 1A, B). The membranes were allowed to form at $23^{\circ} \mathrm{C}$ for $48 \mathrm{~h}$. Membranes were then cross-linked overnight at $37^{\circ} \mathrm{C}$ with $3.15 \mathrm{mM}$ GNP, 1.66 mM SCM-PEG-SCM, $0.87 \mathrm{mM} 4 \mathrm{~S}$-StarPEG (concentration chosen to obtain a ratio $1: 1$ amine groups to cross-linker reactive groups) and $62.50 \mathrm{mM}$ GTA (standard concentration) (Fig. 2). After the cross-linking process, membranes were washed three to five times in ultrapure water to remove excess of cross-linkers from the membranes. 
A Molecules

Sequence

$M w(k D a)$ *

$p I^{* *}$

ELR

PA

MESLLP-[(VPGVG VPGVG VPGEG VPGVG VPGVG) $)_{10^{-}}$ (VGIPG)60 $\left.]_{2}-\left[\text { VPGIG) }{ }_{10} \text {-AVTGRGDSPASS(VPGIG) }\right)_{10}\right]_{2}-\mathrm{V}$

$\mathrm{C}_{15} \mathrm{H}_{31} \mathrm{CONH}$-VVVAAAKKK-CONH${ }_{2}$
112.3

1.1506

3.4

10.3
C-Potential $(m V)^{* * *}$

$-30.5 \pm 4.0$

$67.5 \pm 3.3$

B

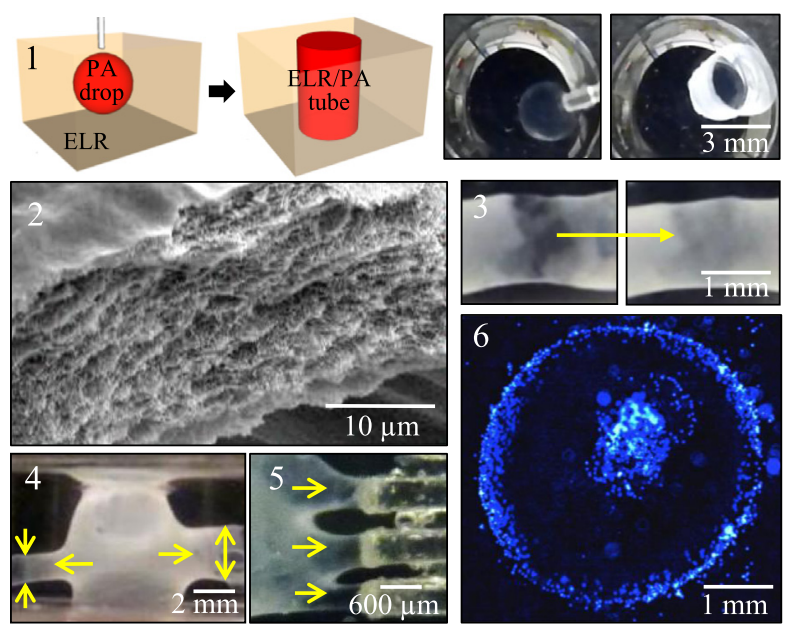

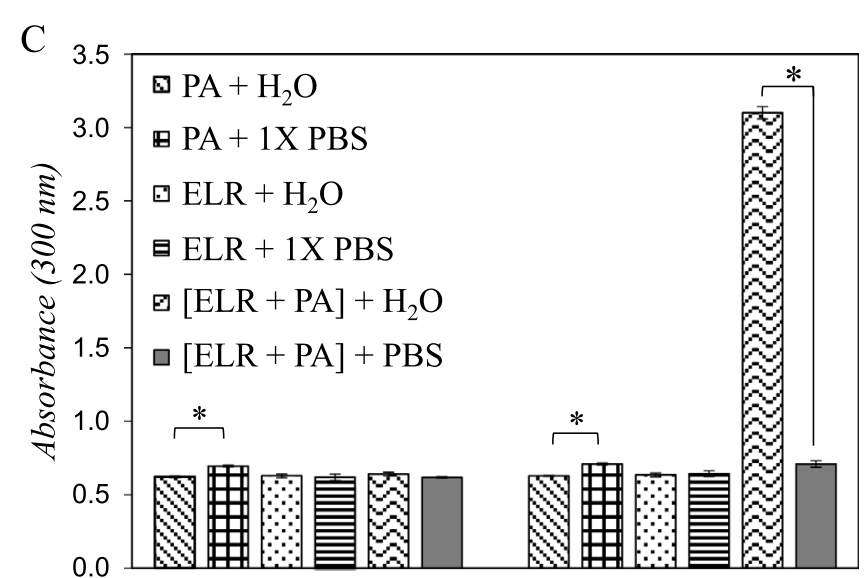

$4^{\circ} \mathrm{C}$

$40^{\circ} \mathrm{C}$

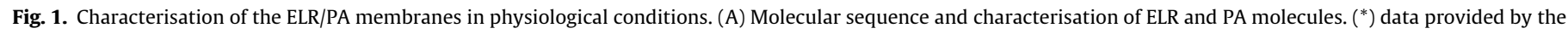

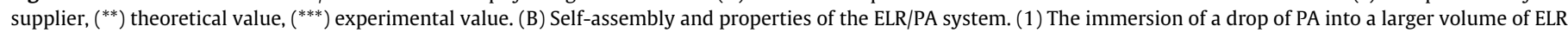

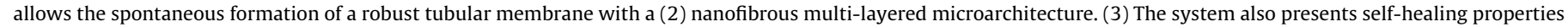

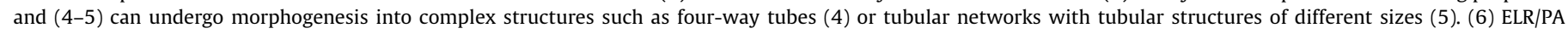

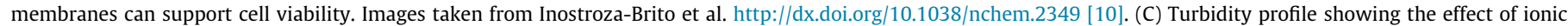

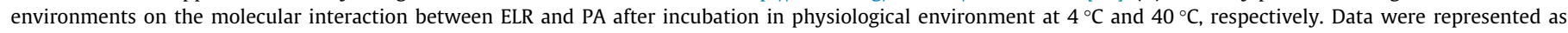
mean \pm standard error of the mean $(n=3)$. ${ }^{*}$ Denotes significant differences between the different groups at $p<0.05$.

\subsection{Effect of physiological solution on ELR/PA aggregates}

The effect of phosphate buffer saline (PBS) on the aggregation process of ELR and PA molecules was evaluated by turbidity assay. Turbidity of the aggregates was measured at 4 and $40^{\circ} \mathrm{C}$ both in water or $1 \times$ PBS. ELR and PA were mixed at lower concentrations ( $0.008 \mathrm{mM}$ and $0.174 \mathrm{mM}$, respectively) than those used to form the membranes in order to avoid formation of visible aggregations or membranes that would have interfered with the measurement. After $5 \mathrm{~min}$ incubation at 4 and $40{ }^{\circ} \mathrm{C}$, absorbance at $300 \mathrm{~nm}$ was measured using an UV-visible spectrophotometer equipped with a temperature controller (SpectrostarNano, BMG Labtech, UK).

\subsection{Effect of physiological solution on ELR/PA membranes}

After fabrication, membranes were rinsed with ultrapure water, cross-linked as previously described, and stored in aqueous solution. Membranes were placed in $1 \times$ PBS at $37{ }^{\circ} \mathrm{C}$ for $48 \mathrm{~h}$. $50 \mu \mathrm{l}$ of $1 \times$ PBS solution were collected after $48 \mathrm{~h}$ for quantifying the free amine groups released in solution. Samples were then prepared for SEM.

\subsection{Scanning electron microscopy (SEM)}

SEM was used to examine the micro and nanoarchitecture of the ELR/PA membranes. Samples were fixed with glutaraldehyde $2.5 \%$ and then dehydrated with serial concentrations of ethanol $(20,50,70,90,96$, and $100 \%)$ prior undergoing critical point drying (K850, Quorum Technologies, UK). Samples were then sputtercoated with gold $(30 \mathrm{~nm})$ and imaged using an Inspect F50 (FEI Comp, The Netherlands).

\subsection{Enzymatic degradation of ER/PA membranes}

The stability of the ELR/PA cross-linked membranes was assessed through enzymatic degradation. Cross-linked membranes were immersed in five enzymatic solutions containing proteinase $\mathrm{K}(\mathrm{PK})$ at $20 \mathrm{U} / \mathrm{mg}$, collagenase $(\mathrm{Cg})$ at $20 \mathrm{U} / \mathrm{mg}$, elastase (Et) at $20 \mathrm{U} / \mathrm{g}$, trypsin (Tp) at $0.05 \mathrm{U} / \mathrm{g}$ and pronase (Pn) at $2 \mathrm{U} / \mathrm{mg}$. Samples were then incubated at $37^{\circ} \mathrm{C}$ for 24 and $48 \mathrm{~h}$ before free amine groups resulting from the proteolytic degradation of the membrane were measured using TNBS assay.

\subsection{Orange II assay and TNBS assay}

The amount of cross-linkage was assessed semi-quantitatively by measuring the amount of free amine groups within the membranes using orange II staining as previously described [24]. Orange II dye has the ability to adsorb onto amine group at low $\mathrm{pH}$ and be released from these groups at high $\mathrm{pH}$ providing an indication of the amount of free amine groups available within the membranes. Briefly, the cross-linked membranes were immersed in $500 \mu$ l of orange II solution $(14 \mathrm{mg} / \mathrm{mL}$; pH 3) for $30 \mathrm{~min}$ at $40{ }^{\circ} \mathrm{C}$. The samples were then intensively washed in acidic water $(\mathrm{pH} 3)$ until all unbounded dye was removed. After air-drying, the samples were immersed in $500 \mu$ l of alkaline solution $(\mathrm{pH}$ 12). After 5 min incubation, the solution containing the desorbed dye was adjusted at $\mathrm{pH} 3$ with a concentrated $\mathrm{HCl}$ solution. Absorbance was then measured at $484 \mathrm{~nm}$ using a microplate reader (SpectroNanoStar, BMG Labtech, UK).

The stability of the membranes was assessed by measurement of released free amine groupsin solution using 2,4,6trinitrobenzenesulfonic acid (TNBS) assay according to manufacturer's instruction. $50 \mu \mathrm{l}$ of $0.01(\mathrm{v} / \mathrm{v}) \%$ TNBS and $75 \mu \mathrm{l}$ of $1 \times$ PBS were then mixed with $25 \mu \mathrm{l}$ from the aliquots previously collected. Mixture was incubated for $2 \mathrm{~h}$ at $37^{\circ} \mathrm{C}$ before addition of $50 \mu \mathrm{l}$ of 
A

\begin{tabular}{c}
$\begin{array}{c}\text { Treatment } \\
\text { group }\end{array}$ \\
\hline Control \\
$4 S-S t a r P E G$ \\
SCM-PEG- \\
SCM \\
GNP \\
GTA
\end{tabular}

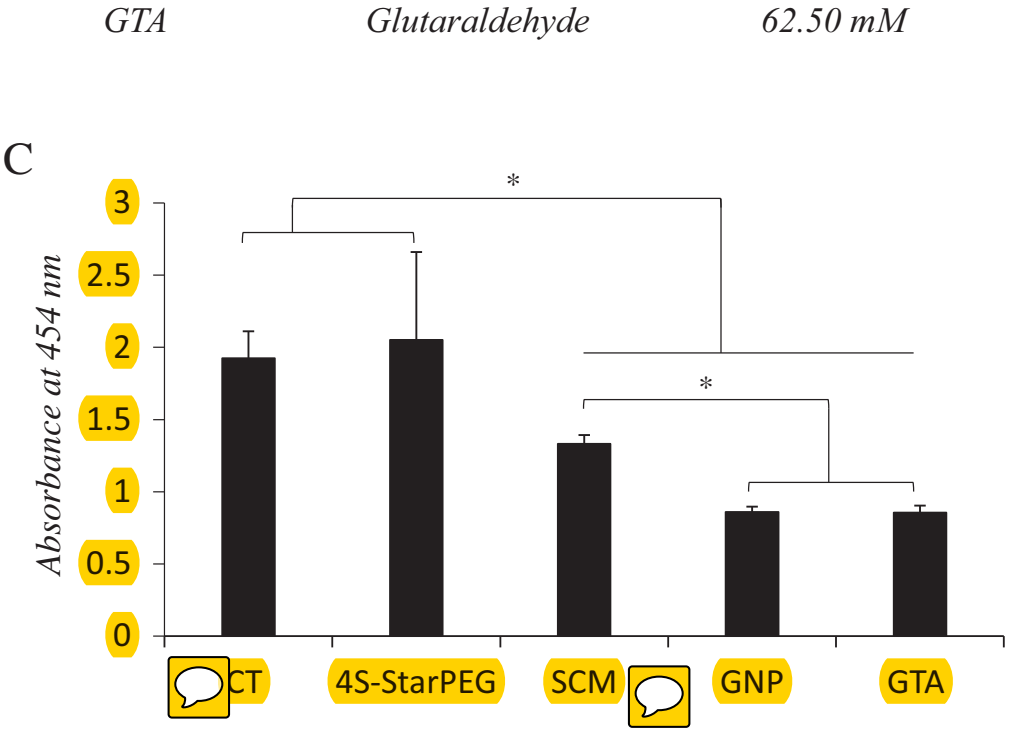

Membranes
Poly (ethylene glycol) ether tetrasuccinimidyl glutarate

Succinimidyl carboxymethyl ester

Genipin

Glutaraldehyde
B

\section{Concentration}

$0 m M$

$0.87 \mathrm{mM}$

$1.66 \mathrm{mM}$

$3.15 \mathrm{mM}$

$62.50 \mathrm{mM}$<smiles>O=CCCCC=O</smiles>

GTA

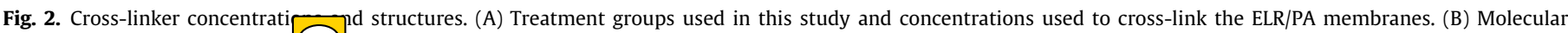

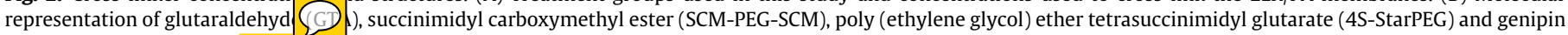

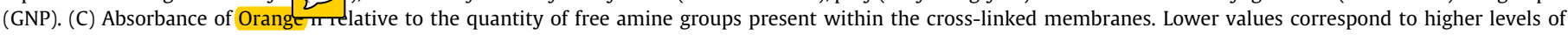
cross-linking $(n=3)$. *Denotes significant differences between the different groups at $p<0.05$.

SDS $10(\mathrm{w} / \mathrm{v}) \%$ and $25 \mu \mathrm{l}$ of $\mathrm{HCl} 1 \mathrm{M}$ in order to stop the TNBS reaction. A standard curve using known concentrations of glycine was used to determine the free amine concentration in solution. Absorbance was measured at $335 \mathrm{~nm}$ using a microplate reader (SpectroNanoStar, BMG Labtech, UK).

\subsection{Mechanical properties by atomic force microscopy (AFM)}

Samples were attached to a petri dish using a drop of cyanoacrylate adhesive, and left for a minute for the adhesive to dry. Samples were then immersed in distilled water. Young's Modulus measurements were taken with a JPK Nanowizard-1 (JPK Instruments, Germany) in force spectroscopy mode, mounted on an inverted optical microscope (IX-81; Olympus, Japan). Quadratic pyramidal cantilevers (MLCT; Bruker, MA, USA) with a spring constant of $0.07 \mathrm{~N} / \mathrm{m}$ and half-angle to face of $17.5^{\circ}$ were used for indentation. The sensitivity of cantilevers was determined before measurements by measuring the slope of the force-distance curve in the AFM software on an empty region of a petri dish. Indentation was carried out with an approach speed of $5 \mu \mathrm{m} / \mathrm{s}$ and a maximum set force of $1 \mathrm{nN}$. Measurements were taken multiple times per region and in multiple regions per sample. The Young's Modulus was calculated by fitting the contact region of the approach curve with the Hertz Contact model [25] using the JPK software, using the above constants and calibrated cantilever sensitivity.

\subsection{Cross-linker toxicity}

All cell experiments were conducted with mouse adipose tissue derived stromal cells (mADSCs). Briefly, mADSCs were extracted from mice adipose tissue harvested from the lower midline area, as described elsewhere [26]. Adipogenic, chondrogenic and osteogenic differentiation assays were conducted to confirm the nature of the extracted cells. Medium (DMEM $1 \times$ supplemented with $10 \%$ FBS and $1 \% \mathrm{P} / \mathrm{S}$ ) was changed every two-three days and cells were maintained sub-confluent before seeding.

\subsection{Direct cross-linker toxicity assay}

50,000 cells $/ \mathrm{cm}^{2}$ were seeded onto tissue culture plate and cultured for $24 \mathrm{~h}$ before treatment. The different cross-linkers were added in the medium onto the cells at the same concentrations used for the cross-linkage of ELR/PA membranes. After $24 \mathrm{~h}$, cell metabolic activity was assessed by AlamarBlue ${ }^{\mathrm{TM}}$ cell metabolic assay (Life Technologies, UK).

\subsection{Cell seeding onto ELR/PA membranes}

Cross-linked membranes were sterilised using UV light for 15 min and washed with Hank's balance solution before cell seeding. 50,000 cells were seeded onto the ELR/PA membranes cross- 
linked as previously described with the different agents. After cell seeding, membranes were incubated for $1 \mathrm{~h}$ under agitation $(150 \mathrm{rpm})$ before culture in static condition for 7 days. mADSCs cultured on tissue culture polystyrene (TCP) were used as control group.

\subsection{Cell metabolic activity}

Cell metabolic activity was assessed using AlamarBlue ${ }^{\mathrm{TM}}$ cell metabolic assay (ThermoFisher Scientific, UK) according to the manufacturer's protocol after 7 days. Briefly, cells were incubated at $37^{\circ} \mathrm{C}$ for $2 \mathrm{~h}$ in a $10 \%$ Alamar Blue solution. Absorbance was read at 570 and $595 \mathrm{~nm}$ using a microplate reader (SpetrostarNano, BMG Labtech, UK). The metabolic activity was calculated according to the simplified method of calculating percentage reduction and normalised by DNA content. DNA quantification was performed using the PicoGreen ${ }^{\circledR}$ assay (ThermoFisher Scientific, UK) as per manufacturer's guidelines. A standard curve of known DNA concentrations was used to determine the DNA content and fluorescence was measured using a microplate reader (FLUOstar Optima, BMG Labtech, UK) at $480 \mathrm{~nm}$ excitation and $520 \mathrm{~nm}$ emission.

\subsection{Cell morphology}

Cell morphology was assessed after iments, samples were fixed with $4 \%$ paraformaldehyde for $1 \mathrm{~h}$. After three washes with $1 \times$ PBS, nucleus and cytoskeleton were stained with DAPI (ThermoFisher Scientific, UK) and phalloidin CruzFluor $^{\mathrm{TM}} 647$ conjugate (Santa Cruz, USA), respectively. Cells were then imaged using a Leica TCS SP2 inverted confocal microscope.

\subsection{Statistical analysis}

Statistical analysis was performed using Minitab 17 (Minitab ${ }^{\circledR}$, Inc, USA). All values are expressed as mean values $(n=3)$ with standard error of the mean (SEM). After the normality was checked, one-way analysis of variance (ANOVA) for multiple comparisons was employed with post hoc test Tukey. When the population did not present a normal distribution, non-parametric statistics Kruskal-Wallis test for multiple comparisons was carried out with post hoc test Dunns. Statistical significance was accepted at $p<0.05$.

\section{Results and discussion}

\subsection{Membrane weakening in physiological environments}

We have recently reported on a dynamic self-assembly system that combines elastin-like recombinamers (ELRs), also known as elastin-like polypeptides (ELPs) and peptide amphiphiles (PA) (Fig. 1A). This system relies on the conformational modification of the ELR molecules by interacting non-covalently (electrostatic and hydrophobic interactions) with the PA molecules [10]. While the membrane formed is stable in water, a weakening of the system is visible when immersed in ionic solutions (e.g. PBS, cell culture media) (Fig. S1, Supplementary information). To elucidate the mechanism behind this dissociation, the effect of ionic solutions on ELR/PA interactions and ELR folding was studied using a turbidity assay. ELRs are synthetic biopolymers that undergo a reversible phase transition at a specific transition temperature (Tt). At temperatures below this Tt, ELR molecules are highly soluble. When the temperature is risen above the $\mathrm{Tt}$, molecules collapse and aggregate due to the dehydration of their hydrophobic domains
[27], generating a change in the turbidity profile of the ELR solution $[28,29]$. We previously showed that the molecular co-assembly of ELR and PA molecules in water above the Tt modifies the turbidity profile of the solution [10]. However, upon addition of an ionic solution (i.e. $1 \times$ PBS), this turbidity profile is significantly reduced, suggesting that the ions present in the solution modify the interaction between ELR and PA molecules (Fig. 1C). These results are in agreement with previous studies demonstrating a change in ELR conformation in the presence of salts [28,29] and consequently explain the observed dissociation of the ELR/PA membrane overtime in $1 \times$ PBS (Fig. S1, Supplementary information). In order to exploit the benefits of this biopolymer-peptide system, we investigated the performance of cross-linking methods to maximize structural stability while maintaining the structural hierarchy, bioactivity, functionality, and biocompatibility of the system.

\subsection{Crosslinking strategies}

Four exogenous cross-linkers frequently used in tissue engineering applications $[13,16-18,23,30,31]$ were used including glutaraldehyde (GTA) [16,17,32], genipin (GNP) [15,22,33], poly (ethylene glycol) ether tetrasuccinimidyl glutarate (4S-PEG $[12,17,30,31]$, and succinimidyl carboxymethyl ester (SCM-PEG SCM) $[29,34]$ (Fig. 2). GTA was included as a positive control at a concentration used in other systems previously reported in the literature $[35,36]$. Non-cross-linked membranes were used as negative controls. The concentrations of 4S-StarPEG, SCM-PEG-SCM and GNP used to stabilise the ELR/PA membranes were calculated to maximize interactions between the amine groups available in the membrane to a ratio $1: 1$ reactive groups to free amine groups (see Experimental Part Section 2.2).

ELR/PA membranes were fabricated as previously reported [10] and their stability assessed after incubation in a physiological environment $(1 \times$ PBS $)$ and enzymatic degradation. Membranes crosslinked with GTA and GNP exhibited a change in colour from white to orange and blue, respectively (Fig. 3A). The cross-linking process with GNP involves an oxygen radical-induced polymerisation that generates the blue colour [22]. In contrast, cross-linking of the membranes with 4S-StarPEG and SCM-PEG-SCM did not induce a change in colour (no aromatic, cycles and radical polymerisation process), but rather resulted in loss of the distinctive membrane stickiness [10]. Lysine-rich segments have been shown to influence the adhesive properties of materials and organisms $[37,38]$. These segments become unavailable upon cross-linkage of the ELR/PA membranes and therefore result in the decreased stickiness of the membrane. A lower quantity of free amine groups was observed after cross-linkage with SCM-PEG-SCM, GNP and GTA cross-linked membranes (Fig. 2C). Interestingly, no difference in orange II released was observed between non-cross-linked membranes and $4 \mathrm{~S}$-StarPEG cross-linked membranes, suggesting an absence or lower ability of 4S-StarPEG to cross-link the membranes (Fig. 2C).

All cross-linked membranes remained stable when immersed in physiological conditions for up to 21 days, except membranes cross-linked with 4S-StarPEG, while negative control membranes disintegrated after a day. There was no qualitative evidence of swelling of the membranes upon immersion in 1X PBS after cross-linking (Fig. 3A, B). Furthermore, the distinctive multilayer structure of the ELR/PA membranes [10] was conserved independently of the cross-linker used (Fig. 3A), suggesting that the introduction of the cross-linking agent did not interfere with the molecular conformation and organization of the membrane. The stability of the membrane was assessed by quantification of free primary amine groups released after immersion in a physiological solution for $48 \mathrm{~h}$. Significantly lower release of free amine groups was observed on membranes after cross-linkage compared to the 
A

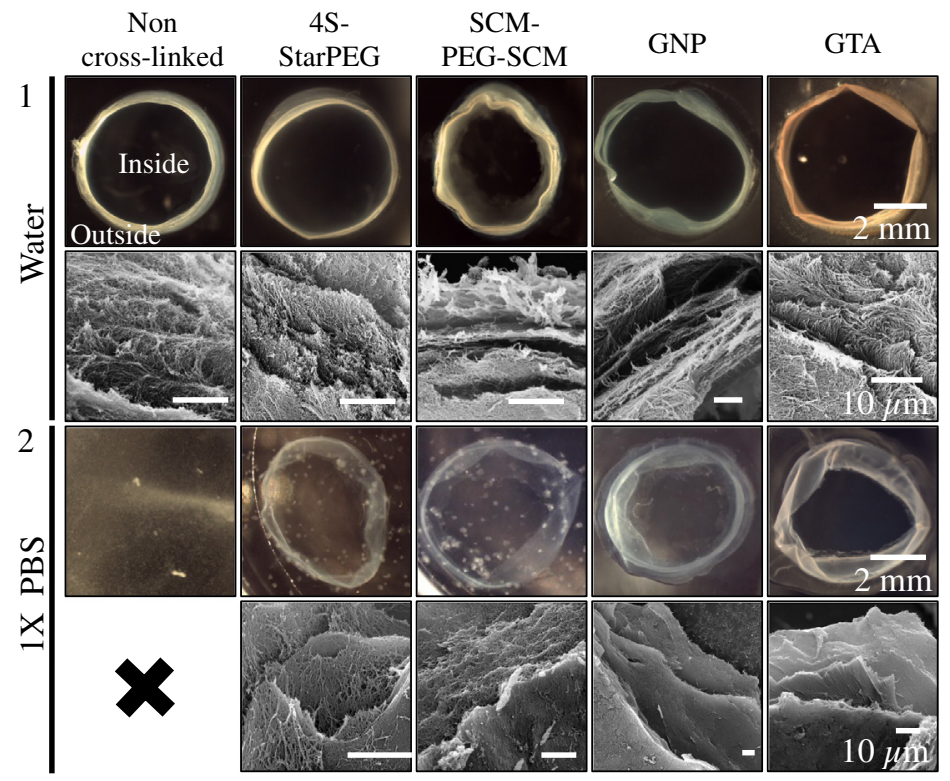

B

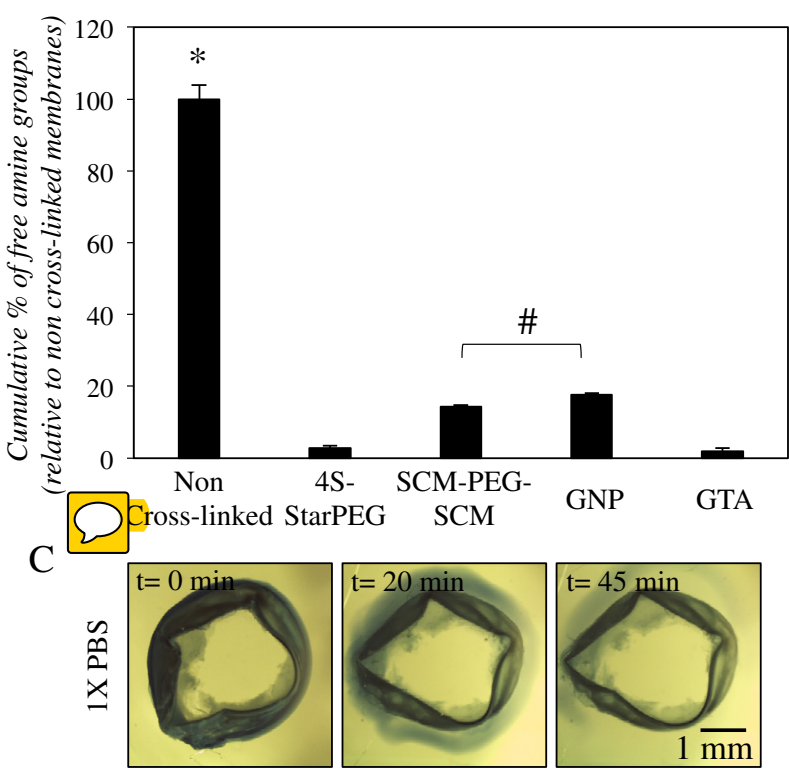

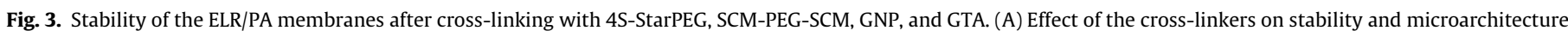

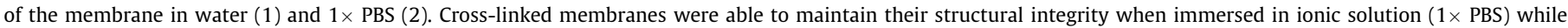

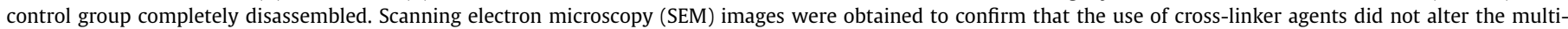

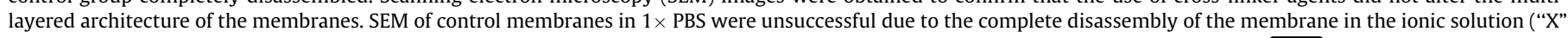

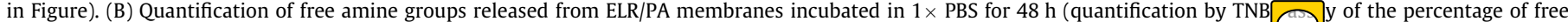

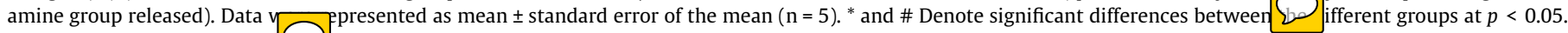

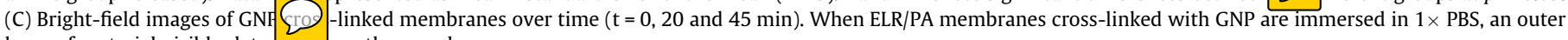
layer of material visibly detacnes nom the membrane.

non-cross-linked membranes $(p<0.05)$ (Fig. 3B), which correlates with the structural modifications observed in non-cross-linked membranes. In contrast, membranes cross-linked with GTA exhibited the lowest amount of free amine groups released followed by those cross-linked with 4 S-StarPEG.

All membranes exhibited an increasing tendency to disintegrate when immersed in ionic solution $(1 \times$ PBS) from 6 to 48 h (GTA reaching $2 \%, 4$ S-StartPEG $4 \%$, and SCM-PEG-SCM $15 \%$ of free amine groups at $48 \mathrm{~h}$ ) except for those cross-linked with GNP (Fig. S2, Supplementary information). In contrast, GNP cross-linked membranes exhibited an initial release of about $18 \%$ of free amine groups, release that stopped after $48 \mathrm{~h}$ of incubation in $1 \times$ PBS (Fig. S2, Supplementary information). All membranes treated with the different cross-linking agents presented an initial loss of material form the outer layer. For example, in membranes cross-linked with GNP, an outer layer of the materials was observed to detach (Fig. 3C). The composition of the outer layer of the membrane was previously reported to be mainly ELR molecules [10]. This loss of amine groups detected by TNBS assay may result from the release of excess ELR weakly bounded to PA on the outer part of the ELR/PA membranes. Nonetheless, all membranes remained stable in physiological conditions $(1 \times \mathrm{PBS})$ after cross-linking (Fig. 3), although membranes cross-linked with 4S-StarPEG exhibited low stability and disassemble after seven days in culture medium (data not shown).

We previously reported the non-equilibrium phase of the ELR/ PA membrane that allows the system to present dynamic properties such as growing, self-healing and morphogenesis (Fig. 1B) [10]. ELR/PA molecules during the non-equilibrium phase are able to self-assemble in membrane shape. However, as we previously reported, this non-equilibrium state can only be maintained in time if i) ELR and PA molecules are available in solution to interact and ii) there is a constant displacement of the surface of the membrane (e.g. displacing the surface of the membrane by adding more
ELR solution into the system) [10]. After acquiring the desired shape and geometry of the ELR/PA membranes, the membranes are incubated for $48 \mathrm{~h}$ to allow them to reach a static-phase, where they are more robust and suitable for further manipulation but dynamic properties are no longer present. The cross-linkage of the membranes was performed after the $48 \mathrm{~h}$ of incubation on membranes without dynamic characteristics due to the previous temporal stabilization of the system. Furthermore, the crosslinkage induces the formation of covalent bonds in the system that prevent the exchange of molecules within the system and therefore its dynamic properties.

\subsection{Stability of the membranes after enzymatic degradation}

In order to provide further insight into the potential performance of the ELR/PA system, we assessed the resistance of the membranes to enzymatic degradation using the proteolytic enzymes collagenase $(\mathrm{Cg})$, proteinase $\mathrm{K}(\mathrm{PK})$, pronase $(\mathrm{Pn})$, trypsin (Tp), and elastase (Et). For this study, we selected the membranes cross-linked with GNP, as this approach enabled the generation of stable membranes with an acceptable degree of structural integrity (Fig. 3A, B) and a low level of cytotoxicity in vitro (Fig. 5). The results demonstrated that GNP cross-linked ELR/PA membranes incubated in proteolytic solutions were susceptible to enzymatic degradation by PK, Pn, Cg, and Et (Fig. 4A). No degradation of the membrane was observed after incubation with Tp (Fig. 4A). TNBS assay revealed a significant increase in free amine groups released after exposure to $\mathrm{PK}, \mathrm{Pn}, \mathrm{Cg}$ and $\mathrm{Et}$, while lower degradation rates were observed for membranes treated with Tp (Fig. 4B). This difference in degradation susceptibility can be explained by the ELR amino acid sequence. ELRs are biopolymers composed of repetitions of the pentapeptide motif Val-Pro-Gly-Xaa-Gly, where Xaa can be any amino acid except proline [39] and have been shown to be susceptible to degradation upon exposure to collagenase 
A

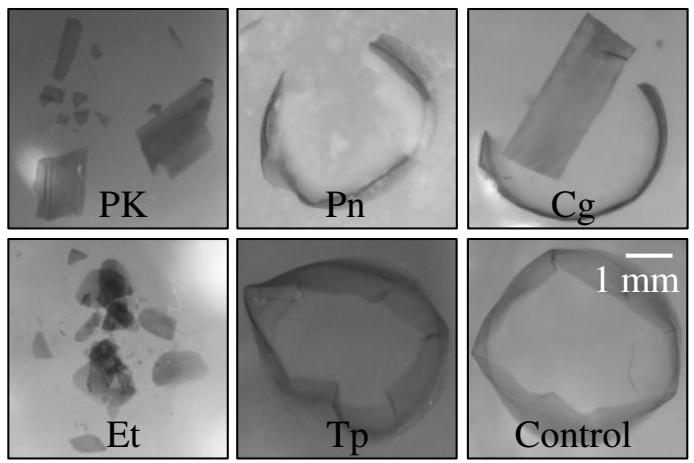

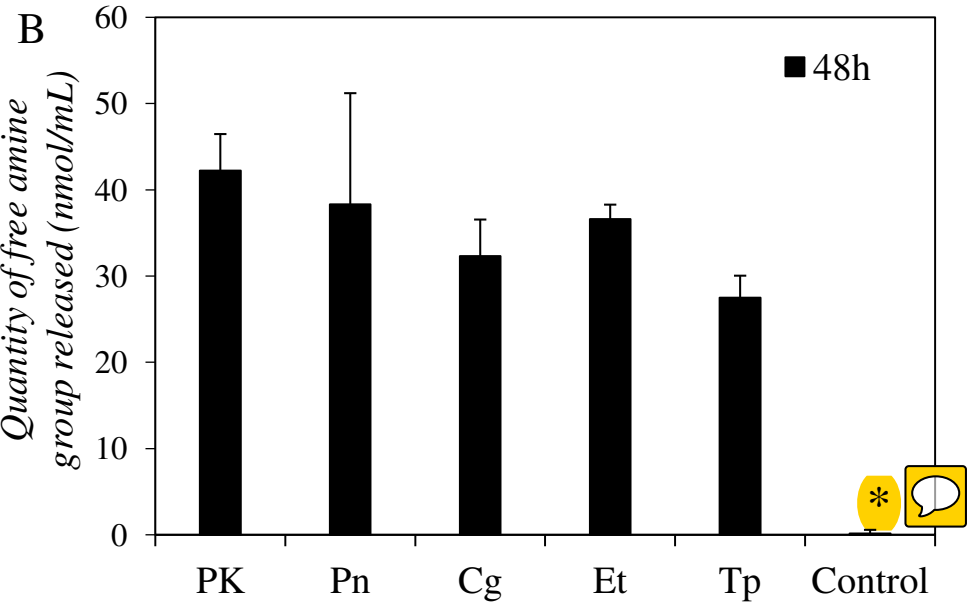

$\mathrm{C}$

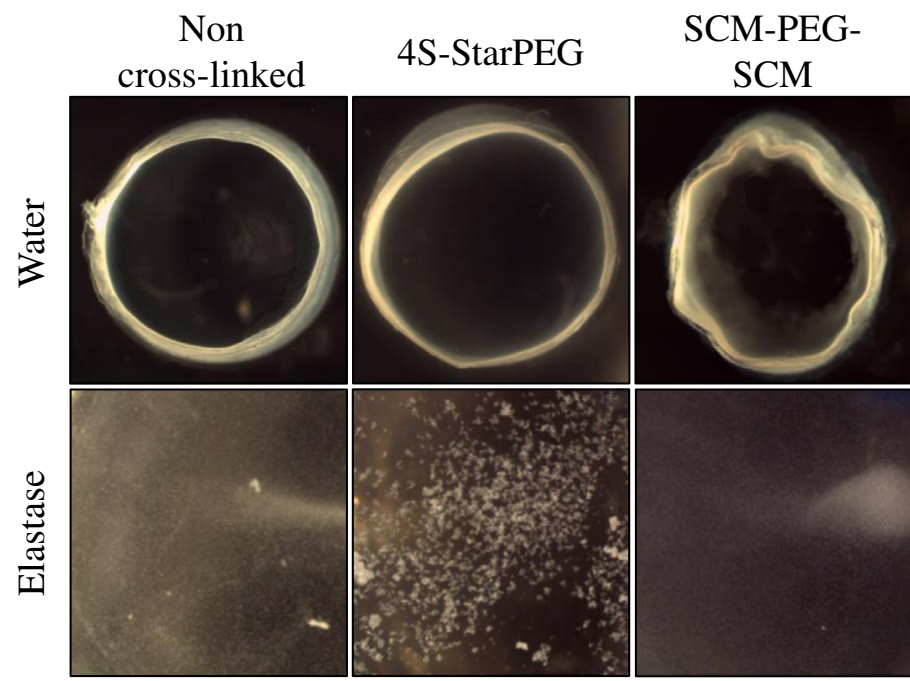

SCM-PEGSCM

GNP

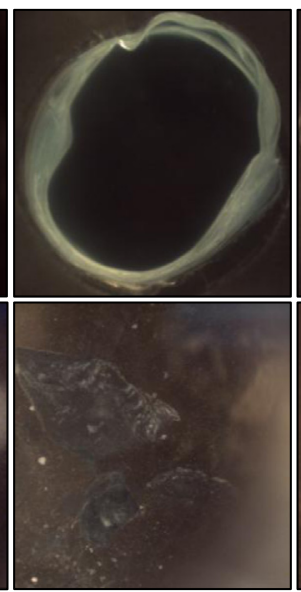

GTA

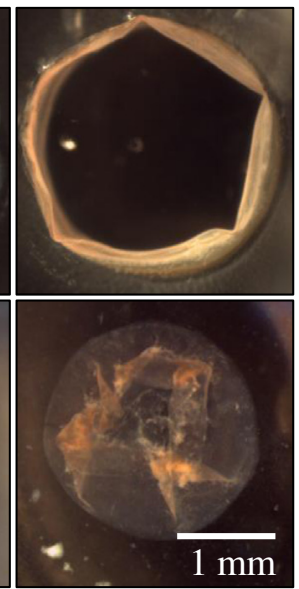

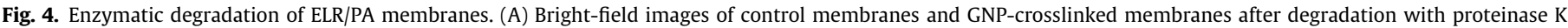

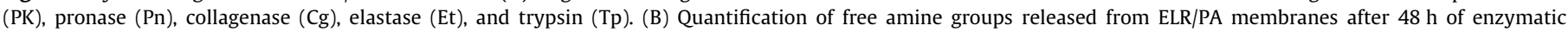

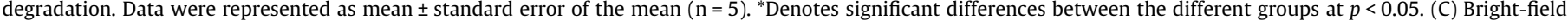

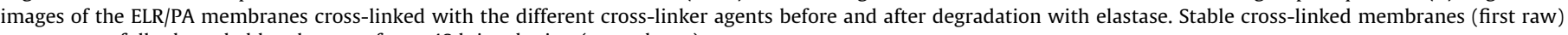
were successfully degraded by elastase after a $48 \mathrm{~h}$ incubation (second raw).

and elastase [40]. Elastase cleavage occurs on the carboxyl end of small and neutral hydrophobic amino acids such as Gly and Val, both of which are present in the ELR molecules used in this study [41]. Similarly, collagenase cleavage occurs between Y-Gly bonds where Y can be any amino acid [42]. Proteinase $\mathrm{K}$ and pronase are two broad serine enzymes cleaving preferentially after hydrophobic amino acids [43] while trypsin cleaves exclusively C-terminal arginine and lysine residues [44]. Therefore, the different levels of degradation according to the enzyme used can be attributed to the enzyme cleavage site preference. All enzymes except trypsin were able to degrade to some degree the ELR/PA membranes.

As elastase is the most physiologically relevant enzyme used to degrade ELRs [37], human leucocyte elastase was chosen to further test the efficiency of the cross-linkage process in membranes treated with the different cross-linker agents used in this study. The resistance of the membranes to enzymatic degradation was tested. Cross-linked ELR/PA membranes were incubated with elastase for 24 and $48 \mathrm{~h}$. TNBS assay was conducted, verifying the release of free amine group in solution (Fig. 4C), which confirms the potential biodegradability of the ELR/PA membranes in vivo. The use of different cross-linking agents does not protect the ELR/PA system from degradation by elastase. It is also worth noting that GNP and GTA cross-linked membranes exhibited a lower degradation rate $(p<0.05)$ and higher stability than the other cross-linked membranes (Fig. $4 \mathrm{C}$ ) as remaining of the membrane are still visible in solution after enzymatic degradation.

\subsection{Cytocompatibility of cross-linkers and membranes}

A major objective of the study was to identify the most effective cross-linker with the capacity to improve stability with minimal cytotoxicity. To investigate the cytocompatibility of the tested cross-linkers, mouse adipose derived stem cells (mADSCs) were exposed to the different cross-linkers either in solution while growing on tissue culture plastic (TCP) or after seeding onto cross-linked membranes washed beforehand and culturing for up to seven days.

After direct exposure to the tested cross-linkers, different mADSCs responses were observed. No effect on metabolic activity was observed after adding 4S-StarPEG in contact with mADSCs while those cultured with SCM-PEG-SCM exhibited a 20\% reduction of metabolic activity. The highest reduction in metabolic activity was observed in cells cultured with GNP (55\%) and GTA (60\%) 

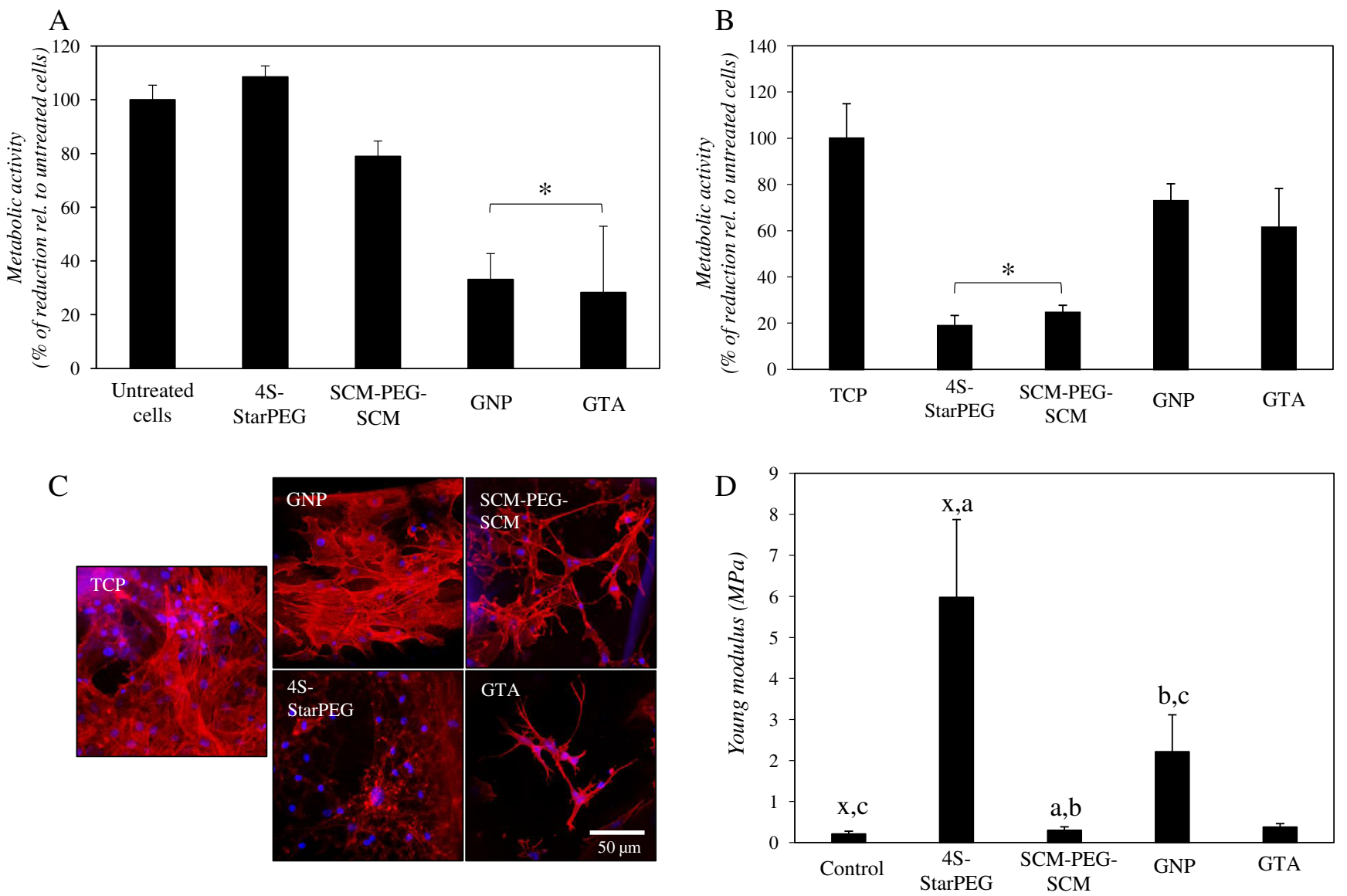

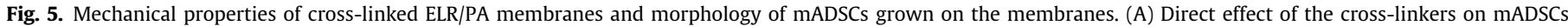

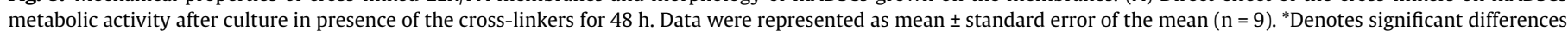

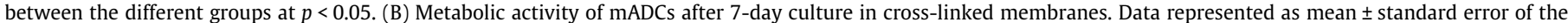

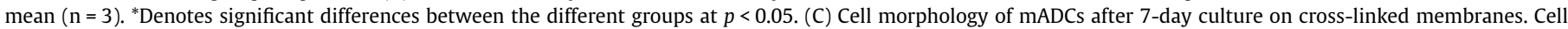

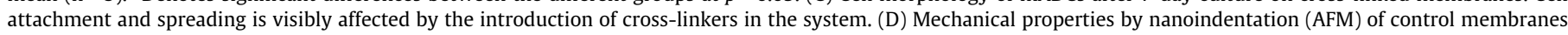

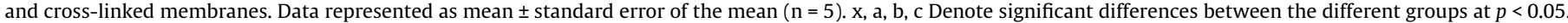

with significant change in metabolic activity compared to that of cells cultured on TCP $(p<0.05)$ (Fig. 5A). This reduction in metabolic activity is associated with a higher degree of cytotoxicity of GNP and GTA as previously reported [45]. Washing steps overcame these issues and allowed cell seeding onto GNP and GTA crosslinked membranes with significantly decreased levels of the cross-linker toxicity (Fig. 5B) [46,47].

Cells seeded onto GNP and GTA cross-linked membranes exhibited the highest metabolic activity without significant difference compared to the metabolic activity of cells cultured on TCP, while a reduction of cell of metabolic activity was observed after culturing on SCM-PEG-SCM $(24.54 \pm 4.44 \%)$ and 4S-StarPEG $(18.87 \pm 7.69 \%)$ crosslinked membranes (Fig. 5C). The observed disassembly of the scaffolds in physiological conditions (Fig. $3 \mathrm{~A}$ ) may increase the toxicity (Fig. 5B) through the release of PA within the medium and/or a modification of the cell attachment sites. PA molecules used to fabricate the ELR/PA membranes were reported to present a certain degree of toxicity [48]. Their release within the medium could therefore induce some cytotoxicity. The crosslinking process could also change the structure of the membrane, limiting the availability of the RGD binding site [49]. Thus, cell attachment would be limited which would result in cell death.

\subsection{Cell morphology}

mADSC adhesion and morphology were assessed after seven days in culture onto ELR/PA membranes cross-linked with GNP,
4S-StarPEG, SCM-PEG-SCM, and GTA, respectively. All membranes remained stable in culture for at least seven days (Fig. 5B) [10]. Differences in cell morphology were observed after culture onto the ELR/PA membranes depending on the cross-linker used for membrane stabilisation (Fig. 5C). Cells cultured on GNP cross-linked membranes displayed a fibroblast-like (elongated spindle) morphology with abundant cytoplasm and large nuclei characteristic of ADSCs [50] and similar to that of observed on TCP (Fig. 5C). In contrast, cells grown on 4S-StarPEG, SCM-PEG-SCM, and GTA cross-linked membranes showed differences in morphology compared to cells cultured on GNP and TCP. Indeed, on 4S-StarPEG, and SCM-PEG-SCM cross-linked membranes, mADSCs conserved a large cytoplasm, although smaller than that of cells cultured on GNP cross-linked membranes, with long filopodia. On the GTA cross-linked membranes, cells exhibited an elongated shape with numerous branching, long, and thin filopodia. The membranes used in this study were fabricated with ELR molecules comprising RGD motifs. This bioactive epitope is the principal integrin-binding domain within ECM proteins such as fibronectin [46]. The presentation of RGD domains to the cells influences cell attachment and spreading [46,51]. Cross-linking processes were previously shown to affect the presentation of RGDS epitopes onto materials [46]. The ELR/PA co-assembled fibres of the membranes may be reorganised during GTA and SCM-PEG-SCM cross-linking processes. This phenomenon could lead to a change in the presentation or availability of the RGD binding sites, which could affect cell attachment and spreading onto these membranes (Fig. 5C). The large size 
of the PEG chains in the 4-StarPEG and SCM-PEG-SCM molecules may hide the RGDS motifs and prevent cell attachments onto the membranes by their antifouling properties [3,52]. Furthermore, a looser mesh of fibres for the non-cross-linked membranes and insufficiently cross-linked (4-StarPEG and SCM-PEG-SCM) membranes could be responsible for this morphology change. Cells may sense the looser and less cross-linked nanofibrous mesh (Fig. S3, Supplementary information) and therefore have difficulties attaching to the structure $[4,53]$.

\subsubsection{Micro-rheological properties of ELR/PA membranes}

It is also likely that the differences in cell attachment and spreading can be a result from differences in surface stiffness $[49,54]$ or roughness [53]. To test this hypothesis, atomic force microscopy (AFM) nanoindentation measurements were conducted to test the mechanical properties of all membranes. Results confirmed a higher stiffness for GNP cross-linked membranes compared to non-cross-linked, SCM-PEG-SCM, and GTA cross-linked membranes $(p<0.05)$ (Fig. 5D). 4S-StarPEG cross-linked membranes were also mechanically stronger. Nanoindentation can be used to measure rheological properties of soft biomaterials [46] such as the ELR/PA membranes developed here. However, the small size of the scanned membranes limit this technique [55]. Here, the area of the scanned sample does not take in consideration the total bulk of the material as only about $1 \mu \mathrm{m}$ was indented into the samples providing only the Young's modulus values of the outer layer of the ELR/PA membranes [56,57]. The ELR/PA membranes exhibit a nanofibrous multi-layered architecture. A lower cross-linking capabilities were observed for the 4S-StarPEG crosslinked membrane (Fig. 2C). As this cross-linking agent has the highest molecular weight ( $10 \mathrm{kDa})$ compared to the other crosslinkers, it is likely that the diffusion of the cross-linker throughout the cross-section of the membrane might have been compromised. Therefore, the cross-linking process may not occur uniformly. The outer layers of the membrane may experience a higher exposure to the cross-linker compared to the inside layers of the membrane. This uneven distribution of the cross-linker exposure may have led to insufficient cross-linking within the membrane, resulting in a higher stiffness on the surface of the membrane (Fig. S3, Supplementary information) and overall weaker membranes more susceptible to disassembly. The stiffness of the membranes stabilized with GTA was significantly enhanced when compare to the control group. However, the use of this specific cross-linker agent led to detrimental effects on cell attachment and spreading (Fig. 5D), highlighting its unsuitability for biomedical applications. In contrast, cells on GNP cross-linked membranes exhibited appropriate attachment, spreading, and proliferation, demonstrating that GNP would be a more effective and cell-friendly cross-linker of self-assembling biomaterials.

\section{Conclusion}

The engineering of more complex biomaterials that can recreate and selectively interact with biological structures would require fabrication processes that can provide both molecular precision and hierarchical organization. Self-assembling systems are at the forefront of this pursuit but require improvement of their structural integrity. Here, we conducted a comparative study to characterize potential cross-linking strategies for self-assembling biomaterials tested on a biopolymer-peptide co-assembly system. The materials were assessed based on their effect on stability, resistance to enzymatic degradation, structural integrity, and biocompatibility. All cross-linkers successfully stabilized the system under physiological conditions but their biocompatibility differed among them. GNP exhibited a higher degree of resistance in physiological environments, improved stability under enzymatic degradation, and a higher degree of in vitro cytocompatibility compared to all other tested. This study provides a systematic comparison of different commonly used cross-linkers and demonstrates that GNP is a promising alternative to improve stability and the structural integrity of self-assembling materials for tissue engineering applications.

\section{Disclosure}

The authors declare no competing financial interests.

\section{Acknowledgements}

This work was supported by the ERC Starting Grant (STROFUNSCAFF and ForceRegulation), The Marie Curie 11 Integration Grant FP7-PEOPLE-2013-CIG (BIOMORPH), NMP-2014-646075 (ELASTISLET), MINECO (MAT2013-42473-R and MAT2015-68901R) and JCyL (VA244U13, VA313U14 and VA015U16). The authors thank the technical staff of the material characterisation and nanovision laboratories at Queen Mary University of London for the constructive discussion and contributions in this study.

\section{Appendix A. Supplementary data}

Supplementary data associated with this article can be found, in the online version, at http://dx.doi.org/10.1016/j.actbio.2017.05. 043.

\section{References}

[1] M.J. Webber, E.A. Appel, E.W. Meijer, R. Langer, Supramolecular biomaterials, Nat. Mater. 15 (1) (2016) 13-26.

[2] E.J. Berns, S. Sur, L. Pan, J.E. Goldberger, S. Suresh, S. Zhang, J.A. Kessler, S.I. Stupp, Aligned neurite outgrowth and directed cell migration in selfassembled monodomain gels, Biomaterials 35 (1) (2014) 185-195.

[3] R.M. Capito, H.S. Azevedo, Y.S. Velichko, A. Mata, S.I. Stupp, Self-assembly of large and small molecules into hierarchically ordered sacs and membranes, Science 319 (5871) (2008) 1812-1816.

[4] G. Fichman, E. Gazit, Self-assembly of short peptides to form hydrogels: design of building blocks, physical properties and technological applications, Acta Biomater. 10 (4) (2014) 1671-1682.

[5] E.R. da Silva, M.N. Walter, M. Reza, V. Castelletto, J. Ruokolainen, C.J. Connon, W.A. Alves, I.W. Hamley, Self-assembled arginine-capped peptide bolaamphiphile nanosheets for cell culture and controlled wettability surfaces, Biomacromolecules 16 (10) (2015) 3180-3190.

[6] L. Adler-Abramovich, E. Gazit, The physical properties of supramolecular peptide assemblies: from building block association to technological applications, Chem. Soc. Rev. 43 (20) (2014) 6881-6893.

[7] C. Tang, A.F. Miller, A. Saiani, Peptide hydrogels as mucoadhesives for local drug delivery, Int. J. Pharm. 465 (1-2) (2014) 427-435.

[8] H. Cui, M.J. Webber, S.I. Stupp, Self-assembly of peptide amphiphiles: from molecules to nanostructures to biomaterials, Biopolymers 94 (1) (2010) 1-18.

[9] G.M. Whitesides, B. Grzybowski, Self-assembly at all scales, Science 295 (5564) (2002) 2418-2421.

[10] K.E. Inostroza-Brito, E. Collin, O. Siton-Mendelson, Co-assembly, spatiotemporal control and morphogenesis of a hybrid protein-peptide system, Nature (2015).

[11] A.C. Mendes, K.H. Smith, E. Tejeda-Montes, E. Engel, R.L. Reis, H.S. Azevedo, A Mata, Co-assembled and microfabricated bioactive membranes, Adv. Funct. Mater. 23 (4) (2013) 430-438.

[12] C.G. Pappas, R. Shafi, I.R. Sasselli, H. Siccardi, T. Wang, V. Narang, R. Abzalimov, N. Wijerathne, R.V. Ulijn, Dynamic peptide libraries for the discovery of supramolecular nanomaterials, Nat. Nanotechnol. 11 (11) (2016) 960-967.

[13] M. Sanami, I. Sweeney, Z. Shtein, S. Meirovich, A. Sorushanova, A.M. Mullen, M. Miraftab, O. Shoseyov, C. O'Dowd, A. Pandit, D.I. Zeugolis, The influence of poly (ethylene glycol) ether tetrasuccinimidyl glutarate on the structural, physical, and biological properties of collagen fibers, J. Biomed. Mater. Res. Part B 104 (5) (2016) 914-922.

[14] J. Li, Z. Suoab, J. Vlassak, Stiff, strong, and tough hydrogels with good chemical stability, J. Mater. Chem. B 2 (2014) 6708-6713.

[15] W.E. Hennink, C.F. van Nostrum, Novel crosslinking methods to design hydrogels, Adv. Drug Deliv. Rev. 54 (1) (2002) 13-36.

[16] Y. Wang, J. Bao, X. Wu, Q. Wu, Y. Li, Y. Zhou, L. Li, H. Bu, Genipin crosslinking reduced the immunogenicity of xenogeneic decellularized porcine whole-liver 
matrices through regulation of immune cell proliferation and polarization, Sci. Rep. 6 (2016) 24779.

[17] H. Saito, S. Murabayashi, Y. Mitamura, T. Taguchi, Characterization of alkalitreated collagen gels prepared by different crosslinkers, J. Mater. Sci. - Mater. Med. 19 (3) (2008) 1297-1305.

[18] E.C. Collin, S. Grad, D.I. Zeugolis, C.S. Vinatier, J.R. Clouet, J.J. Guicheux, P. Weiss, M. Alini, A.S. Pandit, An injectable vehicle for nucleus pulposus cell-based therapy, Biomaterials 32 (11) (2011) 2862-2870.

[19] T. Taguchi, L. Xu, H. Kobayashi, A. Taniguchi, K. Kataoka, J. Tanaka, Encapsulation of chondrocytes in injectable alkali-treated collagen gels prepared using poly(ethylene glycol)-based 4-armed star polymer, Biomaterials 26 (11) (2005) 1247-1252.

[20] G. Nie, H.J. Hah, G. Kim, Y.E. Lee, M. Qin, T.S. Ratani, P. Fotiadis, A. Miller, A. Kochi, D. Gao, T. Chen, D.A. Orringer, O. Sagher, M.A. Philbert, R. Kopelman, Hydrogel nanoparticles with covalently linked coomassie blue for brain tumor delineation visible to the surgeon, Small 8 (6) (2012) 884-891.

[21] L. Solorio, C. Zwolinski, A.W. Lund, M.J. Farrell, J.P. Stegemann, Gelatin microspheres crosslinked with genipin for local delivery of growth factors, J. Tissue Eng. Regen. Med. 4 (7) (2010) 514-523.

[22] V. Chiono, E. Pulieri, G. Vozzi, G. Ciardelli, A. Ahluwalia, P. Giusti, Genipincrosslinked chitosan/gelatin blends for biomedical applications, J. Mater. Sci. Mater. Med. 19 (2) (2008) 889-898.

[23] J.S. Yoo, Y.J. Kim, S.H. Kim, S.H. Choi, Study on genipin: a new alternative natural crosslinking agent for fixing heterograft tissue, Korean J. Thorac. Cardiovasc. Surg. 44 (3) (2011) 197-207.

[24] S. Noel, B. Liberelle, L. Robitaille, G. De Crescenzo, Quantification of primary amine groups available for subsequent biofunctionalization of polymer surfaces, Bioconjug. Chem. 22 (8) (2011) 1690-1699.

[25] A.R. Harris, G.T. Charras, Experimental validation of atomic force microscopybased cell elasticity measurements, Nanotechnology 22 (34) (2011) 345102.

[26] C. Merceron, C. Vinatier, S. Portron, M. Masson, J. Amiaud, L. Guigand, Y. Cherel, P. Weiss, J. Guicheux, Differential effects of hypoxia on osteochondrogenic potential of human adipose-derived stem cells, Am. J. Physiol. Cell Physiol. 298 (2) (2010) C355-64.

[27] D. Urry, Physical chemistry of biological free energy transduction as demonstrated by elastin protein-based polymers, J. Phys. Chem. B 51 (101) (1997) 11007-11028.

[28] T. Christensen, W. Hassouneh, K. Trabbic-Carlson, A. Chilkoti, Predicting transition temperatures of elastin-like polypeptide fusion proteins, Biomacromolecules 14 (5) (2013) 1514-1519.

[29] J.C. Rodriguez-Cabello, S. Prieto, J. Reguera, F.J. Arias, A. Ribeiro, Biofunctional design of elastin-like polymers for advanced applications in nanobiotechnology, J. Biomater. Sci. Polym. Ed. 18 (3) (2007) 269-286.

[30] M. Abu-Rub, S. McMahon, D.I. Zeugolis, A. Windebank, A. Pandit, Spinal cord injury in vitro: modelling axon growth inhibition, Drug Discov. Today 15 (1112) (2010) 436-443.

[31] M. Sanami, Z. Shtein, I. Sweeney, A. Sorushanova, A. Rivkin, M. Miraftab, O. Shoseyov, C. O’Dowd, A.M. Mullen, A. Pandit, D.I. Zeugolis, Biophysical and biological characterisation of collagen/resilin-like protein composite fibres, Biomed. Mater. 10 (6) (2015) 065005.

[32] S. Yu, X. Zhang, G. Tan, L. Tian, D. Liu, Y. Liu, X. Yang, W. Pan, A novel pHinduced thermosensitive hydrogel composed of carboxymethyl chitosan and poloxamer cross-linked by glutaraldehyde for ophthalmic drug delivery, Carbohydr. Polym. 155 (2017) 208-217.

[33] R.A. Muzzarelli, M. El Mehtedi, C. Bottegoni, A. Gigante, Physical properties imparted by genipin to chitosan for tissue regeneration with human stem cells: a review, Int. J. Biol. Macromol. 93 (Pt B) (2016) 1366-1381.

[34] J.M. Walker, J.M. Zaleski, Magnetically triggered radical-generating Fe304 nanoparticles for biopolymer restructuring: application to the extracellular matrix, Chem. Mater. 27 (24) (2015) 8448-8456.

[35] J. Ward, J. Kelly, W. Wang, D.I. Zeugolis, A. Pandit, Amine functionalization of collagen matrices with multifunctional polyethylene glycol systems, Biomacromolecules 11 (11) (2010) 3093-3101.
[36] E. Jorge-Herrero, P. Fernandez, C. Escudero, J.M. Garcia-Paez, J.L. CastilloOlivares, Calcification of pericardial tissue pretreated with different amino acids, Biomaterials 17 (6) (1996) 571-575.

[37] M.T. Buhmann, N. Poulsen, J. Klemm, M.R. Kennedy, C.D. Sherrill, N. Kroger, A tyrosine-rich cell surface protein in the diatom Amphora coffeaeformis identified through transcriptome analysis and genetic transformation, PLoS One 9 (11) (2014) e110369.

[38] J.P. Park, M.J. Choi, S.H. Kim, S.H. Lee, H. Lee, Preparation of sticky Escherichia coli through surface display of an adhesive catecholamine moiety, Appl. Environ. Microbiol. 80 (1) (2014) 43-53.

[39] A. Thapa, W. Han, R.H. Simons, A. Chilkoti, E.Y. Chi, G.P. Lopez, Effect of detergents on the thermal behavior of elastin-like polypeptides, Biopolymers 99 (1) (2013) 55-62.

[40] M. Shah, P.Y. Hsueh, G. Sun, H.Y. Chang, S.M. Janib, J.A. MacKay, Biodegradation of elastin-like polypeptide nanoparticles, Protein Sci. 21 (6) (2012) 743-750.

[41] A.S. Narayanan, R.A. Anwar, The specificity of purified porcine pancreatic elastase, Biochem. J. 114 (1) (1969) 11-17.

[42] M.F. French, K.A. Mookhtiar, H.E. Van Wart, Limited proteolysis of type collagen at hyperreactive sites by class I and II Clostridium histolyticum collagenases: complementary digestion patterns, Biochemistry 26 (3) (1987) 681-687.

[43] W. Ebeling, N. Hennrich, M. Klockow, H. Metz, H.D. Orth, H. Lang, Proteinase K from Tritirachium album Limber, Eur. J. Biochem. 47 (1) (1974) 91-97.

[44] J.V. Olsen, S.E. Ong, M. Mann, Trypsin cleaves exclusively C-terminal to arginine and lysine residues, Mol. Cell. Proteomics 3 (6) (2004) 608-614.

[45] S.N. Rampersad, Multiple applications of Alamar Blue as an indicator of metabolic function and cellular health in cell viability bioassays, Sensors (Basel) 12 (9) (2012) 12347-12360.

[46] S.L. Bellis, Advantages of RGD peptides for directing cell association with biomaterials, Biomaterials 32 (18) (2011) 4205-4210.

[47] L. Calderon, E. Collin, D. Velasco-Bayon, M. Murphy, D. O’Halloran, A. Pandit, Type II collagen-hyaluronan hydrogel-a step towards a scaffold for intervertebral disc tissue engineering, Eur. Cells Mater. 20 (2010) 134-148.

[48] M.J. Webber, J. Tongers, M.A. Renault, J.G. Roncalli, D.W. Losordo, S.I. Stupp, Development of bioactive peptide amphiphiles for therapeutic cell delivery, Acta Biomater. 6 (1) (2010) 3-11.

[49] C.N. Grover, J.H. Gwynne, N. Pugh, S. Hamaia, R.W. Farndale, S.M. Best, R.E. Cameron, Crosslinking and composition influence the surface properties, mechanical stiffness and cell reactivity of collagen-based films, Acta Biomater. 8 (8) (2012) 3080-3090.

[50] J.M. Gimble, A.J. Katz, B.A. Bunnell, Adipose-derived stem cells for regenerative medicine, Circ. Res. 100 (9) (2007) 1249-1260.

[51] V. Castelletto, R.M. Gouveia, C.J. Connon, I.W. Hamley, J. Seitsonen, A. Nykanen, J. Ruokolainen, Alanine-rich amphiphilic peptide containing the RGD cell adhesion motif: a coating material for human fibroblast attachment and culture, Biomater. Sci. 2 (3) (2014) 362-369.

[52] Y. Dang, M. Quan, C.M. Xing, Y.B. Wang, Y.K. Gong, Biocompatible and antifouling coating of cell membrane phosphorylcholine and mussel catechol modified multi-arm PEGs, J. Mater. Chem. B 3 (11) (2015) 2350-2361.

[53] M.A. Alamein, Q. Liu, S. Stephens, S. Skabo, F. Warnke, R. Bourke, P. Heiner, P.H Warnke, Nanospiderwebs: artificial 3D extracellular matrix from nanofibers by novel clinical grade electrospinning for stem cell delivery, Adv. Healthc. Mater. 2 (5) (2013) 702-717.

[54] T. Yeung, P.C. Georges, L.A. Flanagan, B. Marg, M. Ortiz, M. Funaki, N. Zahir, W. Ming, V. Weaver, P.A. Janmey, Effects of substrate stiffness on cell morphology, cytoskeletal structure, and adhesion, Cell. Motil. Cytoskeleton 60 (1) (2005) 24-34.

[55] M.L. Oyen, Nanoindentation of hydrated materials and tissues, Curr. Opin. Solid State Mater. Sci. 19 (16) (2015) 317-323.

[56] E.G. Herbert, P. Sudharshan Phani, K.E. Johanns, Nanoindentation of viscoelastic solids: a critical assessment of experimental methods, Curr. Opin. Solid State Mater. Sci. 19 (6) (2015) 334-339.

[57] A.B. Mann, J.B. Pethica, Nanoindentation studies in a liquid environment, Langmiur 12 (19) (1996) 4583-4586. 